\title{
MHD aspect of current sheet oscillations related to magnetic field gradients
}

\author{
N. V. Erkaev ${ }^{1,2}$, V. S. Semenov ${ }^{3}$, I. V. Kubyshkin ${ }^{3}$, M. V. Kubyshkina ${ }^{3}$, and H. K. Biernat ${ }^{4,5,6}$ \\ ${ }^{1}$ Institute for Computational Modelling, Russian Academy of Sciences, Krasnoyarsk, 660036, Russia \\ ${ }^{2}$ Siberian Federal University, Krasnoyarsk, 660041, Russia \\ ${ }^{3}$ St.Petersburg State University, 198504, Russia \\ ${ }^{4}$ Space Research Institute, Austrian Academy of Sciences, Graz, Austria \\ ${ }^{5}$ Institute for Theoretical Physics, University of Graz, Austria \\ ${ }^{6}$ Institute for Geophysics, Astrophysics, and Meteorology, University of Graz, Austria
}

Received: 2 October 2008 - Revised: 5 December 2008 - Accepted: 15 December 2008 - Published: 23 January 2009

\begin{abstract}
One-fluid ideal MHD model is applied for description of current sheet flapping disturbances appearing due to a gradient of the normal magnetic field component. The wave modes are studied which are associated to the flapping waves observed in the Earth's magnetotail current sheet. In a linear approximation, solutions are obtained for model profiles of the electric current and plasma densities across the current sheet, which are described by hyperbolic functions. The flapping eigenfrequency is found as a function of wave number. For the Earth's magnetotail conditions, the estimated wave group speed is of the order of a few tens kilometers per second. The current sheet can be stable or unstable in dependence on the direction of the gradient of the normal magnetic field component. The obtained dispersion function is used for calculation of the flapping wave disturbances, which are produced by the given initial Gaussian perturbation at the center of the current sheet and propagating towards the flanks. The propagating flapping pulse has a smooth leading front, and a small scale oscillating trailing front, because the short wave oscillations propagate much slower than the long wave ones.
\end{abstract}

Keywords. Magnetospheric physics (Magnetotail; MHD waves and instabilities; Plasma sheet)

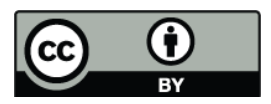

Correspondence to: N. V. Erkaev (erkaev@icm.krasn.ru)

\section{Introduction}

Flapping oscillations of the magnetotail current sheet have been detected by many spacecraft measurements. Namely, CLUSTER observations in the Earth's magnetotail current sheet indicated the appearance of the wave perturbations propagating along the current sheet perpendicular to the magnetic field lines. The observed cases of such waves were first described by Zhang et al. (2002). A comprehensive statistical analysis of Sergeev et al. (2003, 2004), Runov et al. (2005a, b, 2006), and Petrukovich et al. (2006) has proved the existence of such kind of waves, which were identified as the "kink"-like disturbances. The CLUSTER observations are in favor to the assumption, that the flapping perturbations appear more frequently in the central part of the tail, than near the flanks. This statement was also confirmed by Geotail data (Sergeev et al., 2006). In the near-flank tail regions, the flapping waves propagate predominantly from the center to the flanks (Sergeev et al., 2004). These observational results confirm the hypothesis about an internal origin of the flapping motions, due to some nonstationary processes (like magnetic reconnection) localized deep inside the magnetotail. The plasma sheet flapping waves are interpreted as quasi-periodic dynamical structures produced by almost vertical slippage motions of the neighboring magnetic tubes (Petrukovich et al., 2006). Data analysis yields a typical frequency of the flapping waves $w_{f} \sim 0.035 \mathrm{~s}^{-1}$ (Sergeev et al., 2003). A group speed of the flapping waves, estimated from data analysis, is in a range of a few tens (30-70) kilometers per second (Runov et al., 2005a). Spatial amplitudes and wavelengths are of the order of $2-5 R_{E}\left(R_{E}\right.$ is the Earth's

Published by Copernicus Publications on behalf of the European Geosciences Union. 


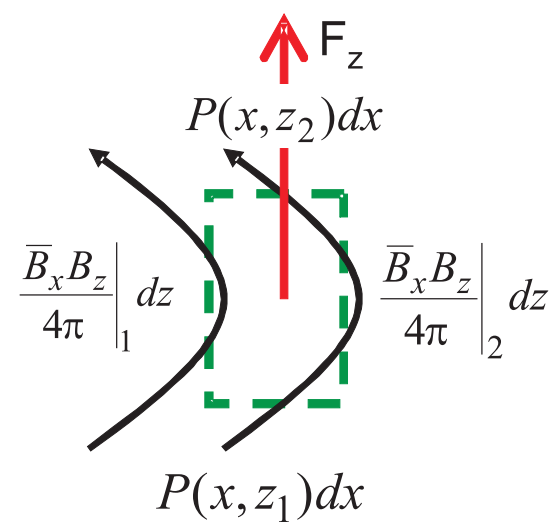

Fig. 1. Displacement of a small element of the current sheet.

radius) (Petrukovich et al., 2006). Using CLUSTER observations, Laitinen et al. (2007) found a relationship between the flapping motions and reconnection events.

In spite of good observational background for the flapping oscillations, a physical mechanism of this phenomenon has not been understood well. Several theoretical models were introduced, but each of them has difficulty in application to this effect. In particular, the Ballooning-type mode was proposed by Golovchanskaya and Maltsev (2005). This ballooning theory implies the condition, that the wave length scale is much less than the curvature radius of the magnetic field line. This condition can hardly be fulfilled in the plasma sheet with a small normal component of the magnetic field.

Volwerk et al. (2003) interpreted flapping oscillations of the current sheet, which were observed before and after substorm onset, as driven magnetoacoustic kink modes studied by Smith et al. (1997). However, the magnetoacoustic waves have too large frequency and wave speed that exceeds the observed values.

Louarn et al. (2004) investigated the low-frequency pressure and magnetic oscillations observed by CLUSTER in the plasma sheet with the aim of determining if they are magnetohydrodynamic (MHD) eigenmodes. This work was further continued by Fruit et al. (2004). They found that the observed kink mode $(0.04 \mathrm{~Hz})$ and the high-frequency sausage mode $(0.14 \mathrm{~Hz})$ are compatible with the MHD model of Harris sheet oscillations propagating in the $\mathrm{x}$-direction, with wavelength of $6 R_{E}$ and $3 R_{E}$.

An interesting physical mechanism addresses to the drift kink modes investigated by Daughton (1999), Karimabadi et al. (2003) and Sitnov et al. (2004). A particular feature of these wave modes is that they can propagate only in the direction determined by the proton drift velocity. However, they can be convected by a bulk flow.

Another theoretical model was proposed by Erkaev et al. $(2007,2008)$ in a framework of MHD approach. In accordance to this model, MHD flapping modes can exist due to a gradient of the normal magnetic field component along the current sheet.
In our present paper, the approach of Erkaev et al. (2007, 2008) is applied for more realistic background current density profile and different plasma density profiles. In addition, we analyze flapping wave propagations towards the flanks from the given sources localized to the center of the current sheet.

\section{Basic equations and model assumptions}

We apply conventional equations of incompressible ideal magnetohydrodynamics (MHD) for nonstationary plasma sheet parameters

$$
\begin{aligned}
& \rho\left(\frac{\partial \mathbf{V}}{\partial t}+\mathbf{V} \cdot \nabla \mathbf{V}\right)+\nabla P=\frac{1}{\mu_{0}} \mathbf{B} \cdot \nabla \mathbf{B}, \\
& \frac{\partial \mathbf{B}}{\partial t}+\mathbf{V} \cdot \nabla \mathbf{B}=\mathbf{B} \cdot \nabla \mathbf{V}, \\
& \frac{\partial \rho}{\partial t}+\mathbf{V} \cdot \nabla \rho=0, \quad \nabla \cdot \mathbf{V}=0, \quad \nabla \cdot \mathbf{B}=0 .
\end{aligned}
$$

Here $\mu_{0}$ is the permeability of free space, $\mathbf{V}, \mathbf{B}, \rho, P$ are the velocity, magnetic field, plasma density and total pressure (sum of the magnetic and plasma pressures), respectively. We consider specific wave perturbations propagating across the magnetic field lines, which are much slower than the magnetosonic modes. In this case the incompressible approximation is quite reasonable. Our study is focussed on the wave modes existing due to a gradient of the $B_{z}$ component in the magnetotail current sheet along the $\mathrm{x}$-direction. Here the $B_{x}$ component has a gradient along the z-direction, and thus we consider the two magnetic gradients as key factors for the current sheet oscillations. This approach, applied by Erkaev et al. (2008) for the flapping wave oscillations, was called as "Magnetic double gradient mechanism".

We begin with a qualitative explanation of the expected MHD flapping instability and waves. A plasma element of a unit volume, considered at the center of the current layer, is shown in Fig. 1. In equilibrium state the total pressure gradient compensates the magnetic tension,

$$
\frac{\partial P}{\partial z}=\frac{1}{\mu_{0}} B_{x} \frac{\partial B_{z}}{\partial x} .
$$

A small displacement of this plasma element along the zdirection yields the restoring force $F_{z}$, which is a difference of two forces caused by the magnetic tension and the total pressure gradient,

$F_{z}=-\frac{1}{\mu_{0}} B_{x}(\delta z) \frac{\partial B_{z}}{\partial x}=-\frac{1}{\mu_{0}} \delta z\left(\frac{\partial B_{x}}{\partial z} \frac{\partial B_{z}}{\partial x}\right)_{z=0}$.

Here $B_{x}(\delta z)$ is determined from the Taylor series expansion. This force accelerates plasma in the z-direction

$\frac{\partial^{2} \delta z}{\partial t^{2}}=\omega_{f}^{2} \delta z$ 
where

$\omega_{f}=\sqrt{\frac{1}{\mu_{0} \rho} \frac{\partial B_{x}}{\partial z} \frac{\partial B_{z}}{\partial x}}$.

In case of positive product of two magnetic gradients, parameter $\omega_{f}$ is real, and it has a meaning of the characteristic frequency of the flapping wave oscillations. In the opposite case of negative product of the magnetic gradients the current sheet is unstable. The flapping perturbations can grow up exponentially without propagation, because $\omega_{f}$ is pure imaginary. These two cases are characterized by different behavior of the background total pressure. Namely, the total pressure has a maximum at the center of the current sheet for the unstable situation, and it has a minimum for the stable conditions. Further we take into consideration MHD Eqs. (13 ) and find solutions corresponding to the qualitative scheme discussed above. The background conditions shown in Fig. 2 are considered to be rather simple with a weak dependence of the $B_{z}$ component on the x-coordinate

$\mathbf{B}=\left[B_{x}(z / \Delta), 0, B_{z}\left(x / L_{x}\right)\right], \quad \mathbf{V}=0$.

Here $\Delta$ is a half-thickness of the current sheet, and $L_{x}$ is a length scale of the $B_{z}$ variation along the current sheet. We introduce two dimensionless parameters $\epsilon=B_{z}(0) / B_{x}$ max and $\nu=\Delta / L_{x}$, which are assumed to be small.

We consider small perturbations of the magnetic field, total pressure and velocity,

$$
\begin{aligned}
& \mathbf{B}=\left(B_{x}+b_{x}, b_{y}, B_{z}+b_{z}\right), \quad \rho=\rho_{0}+\tilde{\rho}, \\
& P=P_{0}+p, \quad \mathbf{V}=\left(v_{x}, v_{y}, v_{z}\right) .
\end{aligned}
$$

As a first step, we make a simplifying assumption, that all wave perturbations propagating in the y-direction do not depend on the $\mathrm{x}$-coordinate, and thus they are considered to be functions of time and two Cartesian coordinates $(y, z)$.

Linearizing Eqs. (1-3) for the small perturbations, we also neglect small terms $B_{z} \nabla_{z} b_{z}$ and $B_{z} \nabla_{z} b_{y}\left(\nabla_{z}\right.$ is a partial derivative with respect to the axis $z$ ), and retain the main term $b_{x} \nabla_{x} B_{z}$. This is justified by the condition $B_{z} L_{x} /\left(B_{x} \Delta\right)=\epsilon / \nu \ll 1$. This condition is valid for the analytical current sheet equilibrium solution of Kan (1973). The linearized equations with the underlined terms to be neglected are the following

$\rho \frac{\partial v_{x}}{\partial t}+\frac{\partial p}{\partial x}=\frac{1}{\mu_{0}}\left(B_{z} \frac{\partial b_{x}}{\partial z}+b_{z} \frac{\partial B_{x}}{\partial z}+B_{x} \frac{\partial b_{x}}{\partial x}+b_{x} \frac{\partial B_{x}}{\partial x}\right)$,

$\rho \frac{\partial v_{y}}{\partial t}+\frac{\partial p}{\partial y}=\frac{1}{\mu_{0}}\left(B_{x} \frac{\partial b_{y}}{\partial x}+B_{z} \frac{\partial b_{y}}{\partial z}\right)$,

$\rho \frac{\partial v_{z}}{\partial t}+\frac{\partial p}{\partial z}=\frac{1}{\mu_{0}}\left(b_{x} \frac{\partial B_{z}}{\partial x}+B_{x} \frac{\partial b_{x}}{\partial x}+B_{z} \frac{\partial b_{z}}{\partial z}+b_{z} \frac{\partial B_{z}}{\partial z}\right)$,

$\frac{\partial b_{x}}{\partial t}+v_{z} \frac{\partial B_{x}}{\partial z}-B_{z} \frac{\partial v_{x}}{\partial z}+\underline{v_{x} \frac{\partial B_{x}}{\partial x}-B_{x} \frac{\partial v_{x}}{\partial x}}=0$,

$\frac{\partial b_{y}}{\partial t}-B_{z} \frac{\partial v_{y}}{\partial z}-\underline{B_{x} \frac{\partial v_{x}}{\partial x}}=0$,

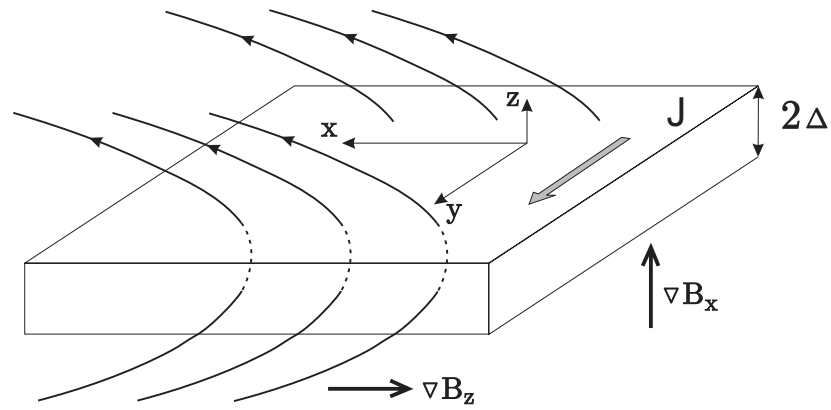

Fig. 2. Geometrical situation of the problem. Background configuration.

$$
\begin{gathered}
\frac{\partial b_{z}}{\partial t}-B_{z} \frac{\partial v_{z}}{\partial z}+v_{x} \frac{\partial B_{z}}{\partial x}-B_{x} \frac{\partial v_{z}}{\partial x}=0, \\
\frac{\partial \rho}{\partial t}+v_{z} \frac{\partial \rho_{0}}{\partial z}=0, \quad \frac{\partial v_{y}}{\partial y}+\frac{\partial v_{z}}{\partial z}=0 .
\end{gathered}
$$

The neglected terms are responsible for the small effects related to the Alfvén waves propagating in the $\mathrm{z}$-direction.

\section{Linear analysis of eigenmodes}

Substituting Fourier harmonics $(\propto \exp (i \omega t-i k y))$ in Eq. (10) and neglecting the underlined terms, we obtain finally a system of equations for Fourier amplitudes

$$
\begin{aligned}
& i \omega \rho_{0} v_{x}=\frac{1}{\mu_{0}}\left(b_{z} \frac{d B_{x}}{d z}+B_{z} \frac{d b_{x}}{d z}\right), \\
& i \omega \rho_{0} v_{y}-i k p=0, \quad i \omega \rho_{0} v_{z}+\frac{d p}{d z}=\frac{1}{\mu_{0}} b_{x} \frac{d B_{z}}{d x}, \\
& i \omega b_{z}-B_{z} \frac{d v_{z}}{d z}+v_{x} \frac{d B_{z}}{d x}=0, \quad i \omega b_{y}-B_{z} \frac{d v_{y}}{d z}=0, \\
& \omega b_{x}+\frac{d B_{x}}{d z} v_{z}=0, \\
& i \omega \tilde{\rho}+v_{z} \frac{d \rho_{0}}{d z}, \quad-i k v_{y}+\frac{d v_{z}}{d z}=0 .
\end{aligned}
$$

Hereafter we assume that gradient $d B_{z} / d x$ is constant, and all other quantities do not depend on the $x$-coordinate. From linearized Eqs. (12-15), treated as a system of ordinary equations with respect to $z$, we derive a second order ordinary differential equation for the velocity perturbation

$$
\frac{1}{\rho_{0}} \frac{d}{d z}\left(\rho_{0} \frac{d v_{z}}{d z}\right)+\bar{k}^{2} \tilde{v}_{z}\left(\frac{U(\bar{z})}{\bar{\omega}^{2}}-1\right)=0,
$$

where

$U(z)=\frac{1}{\mu_{0} \rho_{0}} \frac{\partial B_{x}}{\partial z} \frac{\partial B_{z}}{\partial x}$.

Analytical solution can be obtained for $\rho_{0}=$ const, and a Harris-like variation of the background magnetic field

$B_{x}=B^{*} \tanh (z / \Delta)$. 

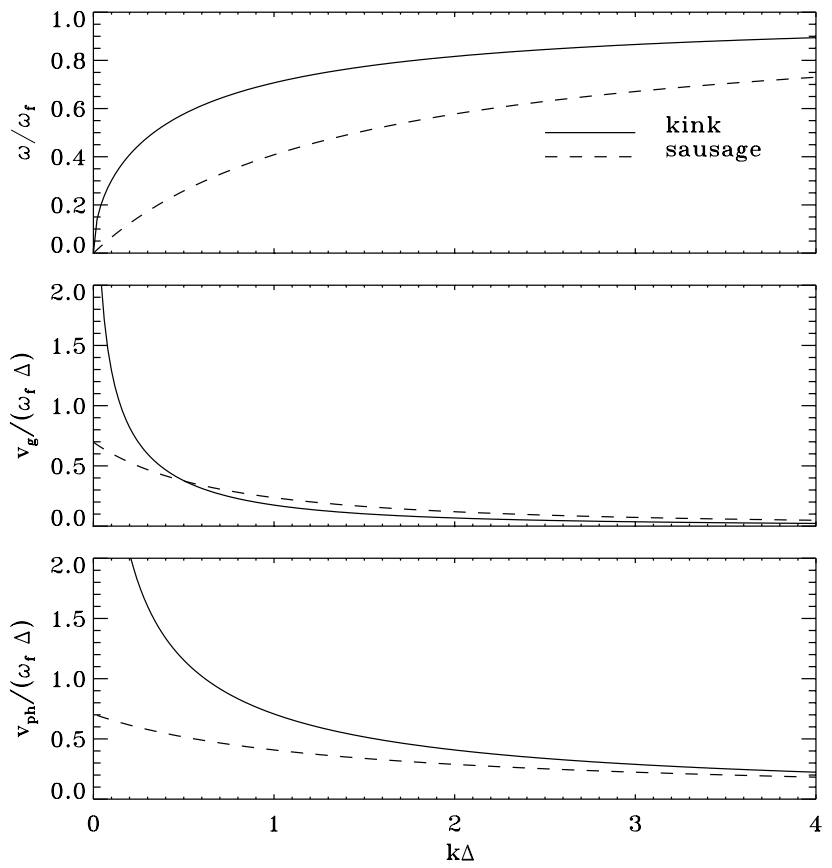

Fig. 3. Frequencies, group and phase velocities as functions of wave number for the "kink" and "sausage" wave modes for the uniform background plasma density.

In this case, Eq. (16) is similar to that known from the theory of tearing mode instabilities (Pritchett et al., 1991). The eigenfunctions are expressed via Legendre functions $\left(P_{\lambda}^{\mu}\right)$ as follows

$$
\begin{aligned}
V_{z} & =C P_{\lambda}^{\mu}(\tanh (z / \Delta)), \\
\lambda & =-1 / 2+\left[1 / 4+\left(k \Delta \omega_{f} / \omega\right)^{2}\right]^{1 / 2}, \\
\mu & =-k \Delta,
\end{aligned}
$$

where

$\omega_{f}=\sqrt{\frac{1}{\mu_{0} \rho} \frac{B^{*}}{\Delta} \frac{\partial B_{z}}{\partial x}}=\sqrt{\frac{1}{\mu_{0} \rho}\left(\frac{\partial B_{x}}{\partial z}\right)_{z=0} \frac{\partial B_{z}}{\partial x}}$.

For the "kink" mode, $V_{z}$ is an even function of the $z$ coordinate, which requires to fulfill condition $\lambda=-\mu=k \Delta$. This relation between $\lambda$ and $k$ yields equation

$k \Delta=-1 / 2+\sqrt{1 / 4+(k \Delta)^{2} \omega_{f}^{2} / \omega^{2}}$.

From this equation we derive frequency and group speed as functions of wave number for the "kink" mode

$$
\begin{aligned}
\omega_{k} & =\omega_{f} \sqrt{\frac{k \Delta}{k \Delta+1},} \\
V_{g k} & =\omega_{f} \Delta F(k \Delta), \\
F(k \Delta) & =\frac{1}{2 \sqrt{k \Delta(1+k \Delta)^{3}}} .
\end{aligned}
$$
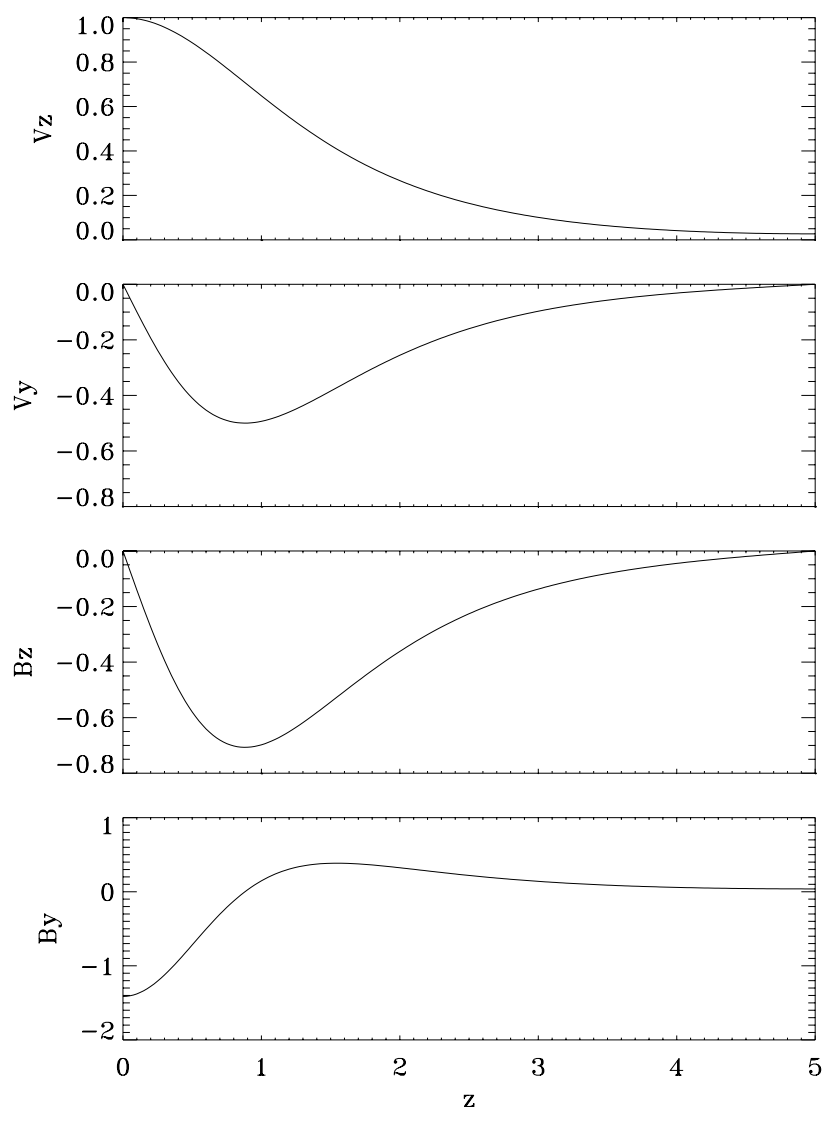

Fig. 4. Eigenfunctions corresponding to the "kink" mode.

For the sausage mode, $V_{z}$ is an odd function, which vanishes at the center of the current sheet. This mode corresponds to condition $\lambda=k \Delta+1$ which leads to

$k \Delta+3 / 2=\sqrt{1 / 4+(k \Delta)^{2} \omega_{f}^{2} / \omega^{2}}$.

This equation determines the "sausage" mode frequency and group speed as an explicit function of wave number

$$
\begin{aligned}
\omega_{s} & =\omega_{f} \frac{k \Delta}{\sqrt{(k \Delta)^{2}+3 k \Delta+2}}, \\
V_{g s} & =\omega_{f} \Delta \frac{3 k \Delta+4}{2 \sqrt{\left[(k \Delta)^{2}+3(k \Delta)+2\right]^{3}}} .
\end{aligned}
$$

The normalized frequencies $\omega_{k, s} / \omega_{f}$ and wave group velocities are shown in Fig. 3. One can see at the figure that the flapping wave frequency is an increasing function of the wave number. It has saturation for short wave length perturbations, which propagate much slower than those with long wave length.

Figure 4 shows the amplitudes of the "kink"-like perturbations of the velocity and magnetic field components as eigenfunctions of z-coordinate for a fixed wave number, $k=\Delta^{-1}$. All perturbations have a smooth behavior, and they vanish far away from the current sheet center. Figure 5 shows the same quantities as Fig. 4, but for the "sausage"-like oscillations. 

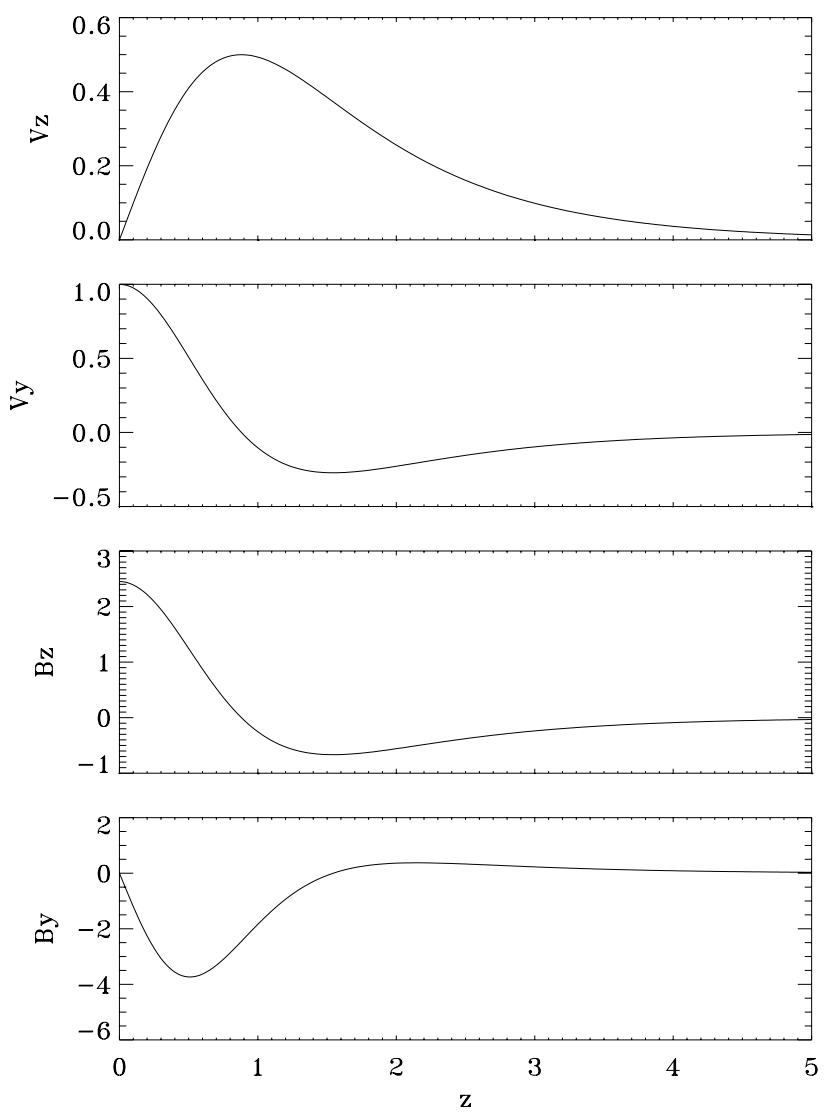

Fig. 5. Eigenfunctions corresponding to the "sausage" mode.

The flapping wave perturbations are unstable, when the product of two magnetic gradients is negative. In this case the flapping frequency is pure imaginary, and the growth times $\tau_{k}$ and $\tau_{s}$ of the instability for the "kink" and "sausage" modes are given by formulas

$\tau_{k}=\tau_{f} \sqrt{1+1 /(k \Delta)}$,

$\tau_{s}=\tau_{f} \sqrt{1+3 /(k \Delta)+2 /(k \Delta)^{2}}$,

$\tau_{f}=\left(\frac{-1}{\mu_{0} \rho} \frac{\partial B_{x}}{\partial z} \frac{\partial B_{z}}{\partial x}\right)^{(-1 / 2)}$.

The "kink" perturbations grow faster than the "sausage" ones. This instability can take place at some regions of the Earth's magnetotail current sheet, where the $B_{z}$ component decreases towards Earth.

For example, we estimate the flapping frequency for the reasonable parameters corresponding to the current sheet conditions in the Earth's magnetotail,

$$
\begin{aligned}
B_{x} & =20 \mathrm{nT}, B_{z}=2 \mathrm{nT}, \Delta \sim R_{E}, n_{p}=0.1 \mathrm{~cm}^{-3}, \\
k \Delta & =0.7, \partial B_{z} / \partial x \sim B_{z} / L_{x}, L_{x} \sim 5 R_{E} .
\end{aligned}
$$

Applying these parameters to Fig. 3, we find the characteristic flapping frequency $\omega_{f} \sim 0.03 \mathrm{~s}^{-1}$, and also the group velocity $V_{g}=60 \mathrm{~km} / \mathrm{s}$, and phase velocity $V_{p h}=274 \mathrm{~km} / \mathrm{s}$.
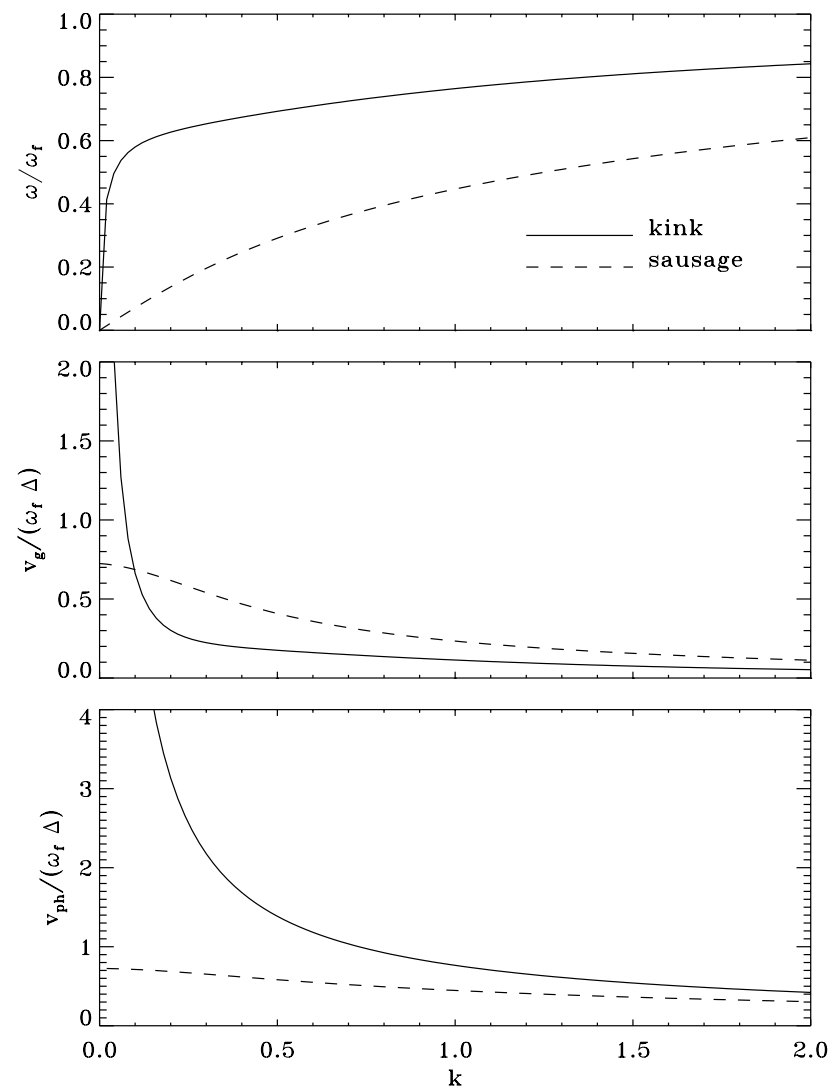

Fig. 6. Frequencies, group and phase velocities in the case of nonconstant density profile.

To estimate the effects related to variations of the background plasma density, we also consider model density function

$\rho_{0}(z)=\rho_{0}^{*} \frac{1}{[\cosh (\eta z / \Delta)]^{2}}$.

In this case we found numerical solution of the boundary eigenvalue problem, and result for $\eta=0.4$ is presented in Fig. 6 that shows the frequency, group and phase velocities as functions of wave number. One can see that the density variation effect is pronounced quite strongly for low wave numbers, and it is rather small for large wave numbers. For two cases, $\eta=0$ and $\eta=0.4$, dispersion functions are rather close to each other, if the wave number sufficiently large: $k \Delta>1$. A noticeable difference between eigenfrequencies appears only for very small wave numbers, $k \Delta \ll 1$. A constant density model $(\eta=0)$ predicts somewhat lower frequency than that for nonhomogeneous density model $(\eta=0.4)$.

\section{Propagation of flapping waves produced by a local- ized source}

The dispersion function obtained in the previous section can be applied for the problem of the flapping wave propagation 


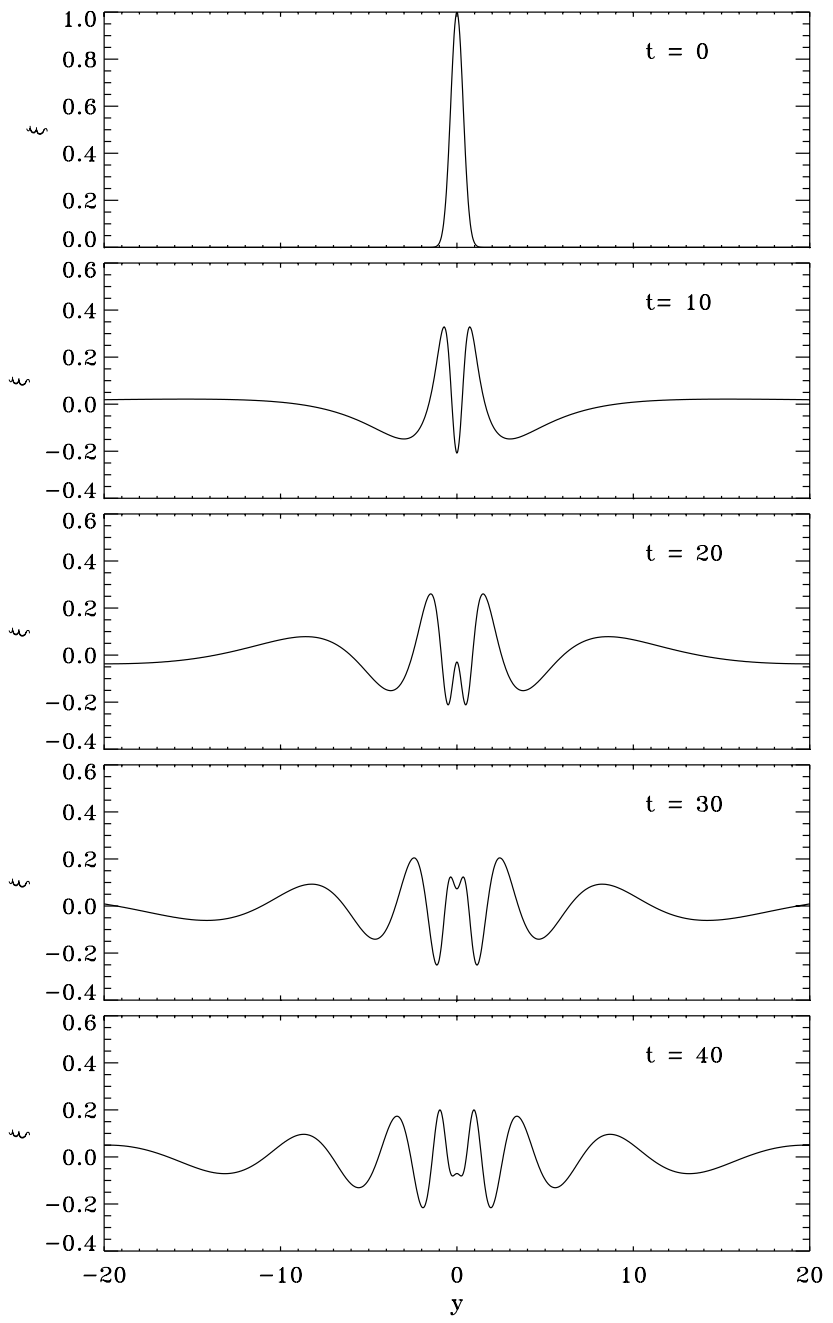

Fig. 7. Flapping waves propagating from the source localized to the center of the current sheet.

from a given source. In particular, we consider the initial "kink"-like Gaussian perturbation $\xi$ of the current sheet which is produced by an external source

$\xi(y, 0)=\xi_{0} \exp \left(-\sigma y^{2}\right)$,

where $\xi_{0}$ and $\sigma$ are the parameters characterizing the amplitude and length scale of the initial disturbance, respectively.

Using Fourier method and dispersion Eq. (22), we find solution for the initial condition (32)

$\xi(y, t)=\int_{-\infty}^{\infty} A(k) \exp [i(\omega(k) t-k y)] d k$,

where function $A(k)$ is given by Fourier integral

$A(k)=\frac{1}{2 \pi} \int_{-\infty}^{\infty} \xi(y, 0) \exp (i k y) d y$.

Figure 7 shows the "kink"-like wave perturbations induced by source (32) as functions of the y-coordinates (in units of

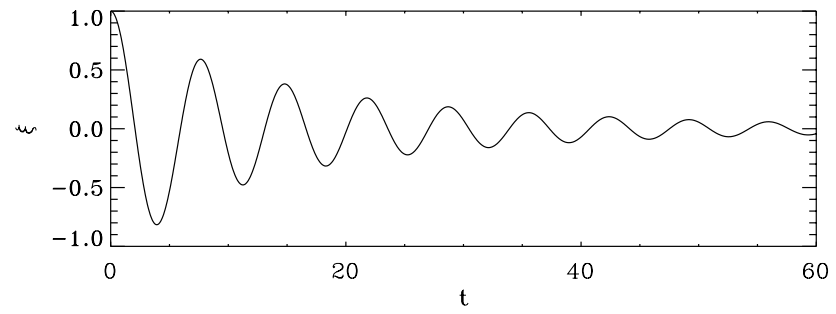

Fig. 8. Oscillations induced by the fixed source localized to the center of the current sheet.

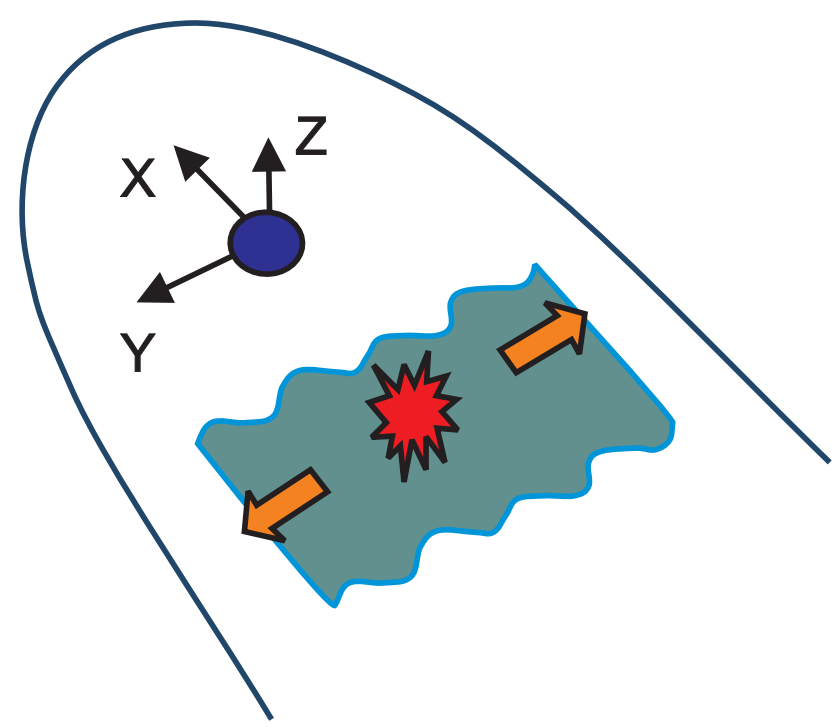

Fig. 9. Position of the source for the flapping waves.

$\Delta$ ) for different times normalized to $\omega_{f}^{-1}$. Figure 8 demonstrates the "kink"-like current sheet oscillations at the point of the initial Gaussian perturbation (at the center of the current sheet) as a function of time. One can see that these oscillations have a rather weak damping. Figure 9 indicates the position of the initial perturbation, which is a source for the flapping wave oscillations (Sergeev et al., 2006). Figure 10 shows perturbations of the magnetic surfaces in the current sheet in cases of the "kink"-like and "sausage"-like flapping oscillations. These wave oscillations, produced by the initial perturbation (Eq. 32), propagate towards the flanks of the current sheet. Next we consider the flapping wave disturbances produced by a moving source, which have to be bounded by a cone with angle $\theta$

$\theta=\arctan \left(1 / M_{f}\right)$,

where $M_{f}$ is the flapping Mach number determined as a ratio of the source velocity $\left(V_{S}\right)$ to the characteristic wave propagation speed.

$M_{f}=\frac{V_{s}}{V_{f}}$, 


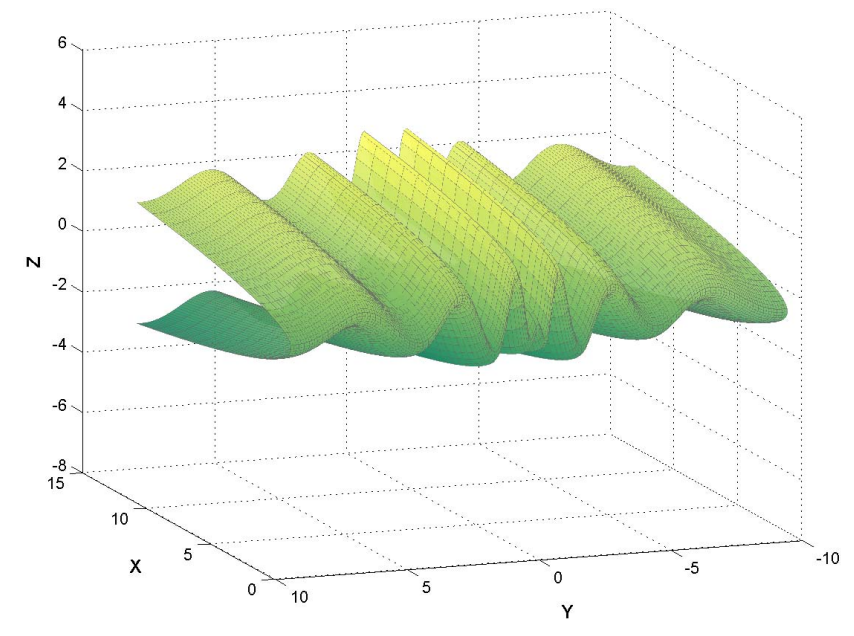

Fig. 10. The "kink"-like disturbances of the current sheet produced by the fixed source.

where $V_{f}$ is the flapping wave group velocity. This is an important dimensionless similarity parameter for flapping waves (like Alfvén and sonic Mach numbers for magnetosonic waves), which characterizes the interaction between moving source and flapping waves in the current sheet.

Figure 11 demonstrates the wave disturbances of the current sheet, which are initiated by a source rapidly moving towards the Earth (hypothetically BBF). From top to bottom, there are shown three pictures corresponding to different normalized velocities (in units of $w_{f} \Delta$ ) of the moving source. Taking reasonable parameters $V_{0} \sim 400 \mathrm{~km} / \mathrm{s}, V_{g} \sim 60 \mathrm{~km} / \mathrm{s}$, we estimate flapping Mach number about $M_{f}=V_{0} / V_{g} \sim 7$. This means that the real flapping cone angle for typical BBFs should be rather small.

\section{Summary}

In a framework of the conventional MHD approach, flapping waves and instability are analyzed in application to the magnetotail current sheet. An important necessary condition for our theory is a presence of the normal magnetic field component $\left(B_{z}\right)$, which has a weak variation along the $\mathrm{x}$-direction. Analytical MHD solution is obtained for the Harris-like current sheet. Dispersion functions are determined for "kink" and "sausage" modes. The frequency and the growth rate for the "kink" mode are found to be much larger than those for the "sausage" mode. For both modes, the frequencies are monotonic increasing functions of the wave number. The corresponding wave group velocities are decreasing functions of the wave number, and they vanish asymptotically for high wave numbers.

Two magnetic gradients, $\nabla_{z} B_{x}$ and $\nabla_{x} B_{z}$, play a crucial role for the stability of the current sheet. It can be stable or unstable, if the product of these magnetic gradients is posi-
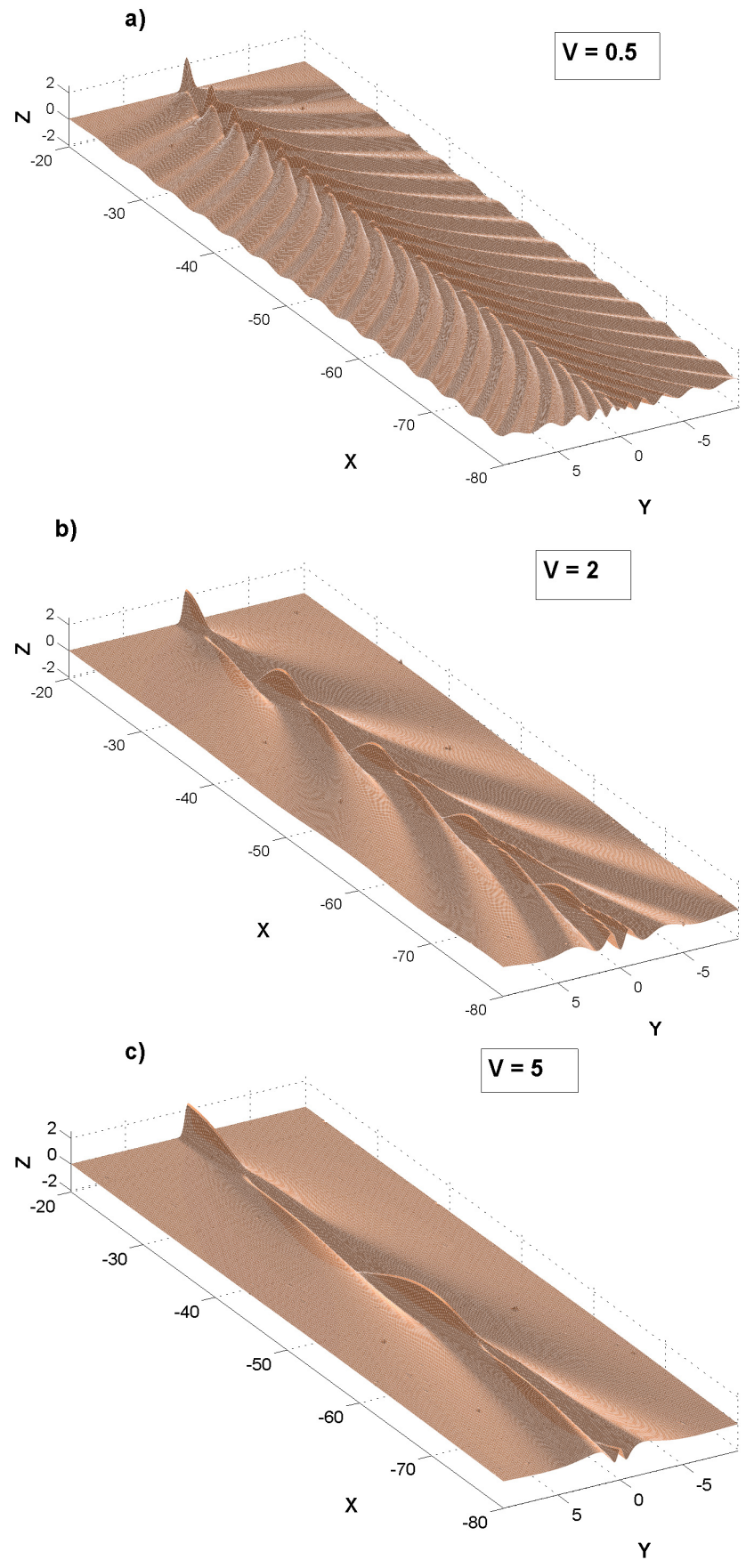

Fig. 11. The "kink"-like disturbances of the current sheet produced by the moving source for different normalized velocities.

tive or negative. In particular, the instability can arise in a vicinity of a localized thinning of the current sheet, where the $B_{z}$ component has inverse gradient. In this unstable situation, the frequency is pure imaginary, and the flapping perturbations just grow-up without propagation. This means that initial static equilibrium state can be destroyed by any small perturbations of the magnetic field and plasma parameters. 
These perturbations will grow up exponentially during time. A nonlinear stabilization might be possible when the displacement amplitude will be of the order of the current sheet thickness

For the typical parameters of the Earth's current sheet, the group velocity of the "kink"-like mode is estimated as a few tens of kilometers per second that is in good agreement with the CLUSTER observations. A strong decrease of the group velocity for high wave numbers means that the small scale oscillations propagate much slower than the large scale oscillations. Because of that, the propagating flapping pulse is expected to have a smooth leading front, and a small scale oscillating trailing front.

The double gradient flapping waves studied in our model propagate in the direction perpendicular to the planes of the background magnetic field lines, and thus they can not be stabilized by the magnetic tension. For the "kink" mode, the magnetic field planes are just shifting with respect to each other.

In the stable region, the flapping oscillations may be initiated by "Bursty Bulk Flows" (BBFs) related to magnetic reconnection pulses in the magnetotail. Existence of such accelerated BBFs is well known from Angelopoulos et al. (1992). These BBFs can induce flapping wave disturbances of the current sheet, like a ship moving on a water surface. Our model predicts also oscillations behind the moving source, which have a very weak damping. The flapping wave disturbances produced by the moving source, has to be bounded by the Mach cone depending on the special flapping Mach number defined as a ratio of the source velocity to the characteristic wave propagation speed. This is the dimensionless similarity parameter for the flapping waves, which characterizes the interaction between moving source and flapping waves in the current sheet. For the realistic simulation of the flapping oscillations produced by BBFs, moving along the magnetotail current sheet, a further development of our approach is required on a basis of threedimensional nonlinear MHD model. Elaboration of such model is a task for future investigation.

Acknowledgements. We thank V. Sergeev for fruitful discussions and help in preparation of the manuscript. This work is supported by RFBR grants N 07-05-00776-a, N 07-05-00135, by SFU grant $\mathrm{N}$ 10, by Programs 2.16 and 16.3 of RAS, and by project P20341-N16 from the Austrian "Fonds zur Förderung der wissenschaftlichen Forschung", and also by project I.2/04 from "Österreichischer Austauschdienst".

Topical Editor R. Nakamura thanks two anonymous referees for their help in evaluating this paper.

\section{References}

Angelopoulos, V., Baumjohann, W., Kennel, C. F., Coroniti, F. V., Kivelson, M. G., Pellat, R., Walker, R. J., Luhr, H., and Paschmann, G.: Bursty bulk flows in the inner central plasma sheet, J. Geophys. Res., 97, 4027-4039, 1992.
Daughton, W.: Two-fluid theory of the drift kink instability, J. Geophys. Res., 104, 28701-28707, 1999.

Erkaev, N. V., Semenov, V. S., and Biernat, H. K.: Magnetic double gradient mechanism for flapping oscillations of a current sheet, Geophys. Res. Lett., 35, L02111, doi:10.1029/2007GL032277, 2008.

Erkaev, N. V., Semenov, V. S., and Biernat H. K.: Magnetic doublegradient instability and flapping waves in a current sheet, Phys. Rev. Lett., 99, 235003, doi:10.1103/PhysRevLett.99.235003, 2007.

Fruit G., Louarn, P., Budnik, P. E., Sauvaud, J. A., Jacquey, C., Réme, H., Lucek, E., Balogh, A., and Cornilleau-Werhlin, N.: Analysis of low-frequency fluctuations in the plamasheet: Signal reconstruction and comparison with MHD theory, J. Geophys. Res., 109, A03217, doi:10.1029/2003JA010229, 2004.

Golovchanskaya, I. V. and Maltsev, Y. P.: On the identification of plasma sheet flapping waves observed by Cluster, Geophys. Res. Lett., 32, L02102, doi:10.1029/2004GL021552, 2005.

Kan, J. R.: On the structure of the magnetotail current sheet, J. Geophys. Res., 78, 3773-3781, 1973.

Karimabadi, H., Daughton, W., Pritchett, P. L., and Krauss-Varban, D.: Ion-ion kink instability in the magnetotail. Linear theory, J. Geophys. Res., 108(A11), 1400, doi:10.1029/2003JA010026, 2003.

Karimabadi, H., Pritchett, P. L., Daughton, W., and KraussVarban, D.: Ion-ion kink instability in the magnetotail. Threedimensional full particle and hybrid simulations and comparison with observations, J. Geophys. Res., 108(A11), 1401, doi:10.1029/2003JA010109, 2003.

Laitinen, T. V., Nakamura, R., Runov, A., Rème, H., and Lucek, E. A.: Global and local disturbances in the magnetotail during reconnection, Ann. Geophys., 25, 1025-1035, 2007, http://www.ann-geophys.net/25/1025/2007/.

Louarn, P., Fruit, G., Budnik, E., Sauvaud, J. A., Jacquey, C., Réme, H., Lucek, E., Balogh, A., and Cornilleau-Werhlin, N.: Analysis of low-frequency fluctuations in the plamasheet: CLUSTER observations and magnetohydrodynamic analysis, J. Geophys. Res., 109, A03216, doi:10.1029/2003JA010228, 2004.

Petrukovich, A. A., Zhang, T. 1., Baumjohann, W., Nakamura, R., Runov, A., Balogh, A., and Carr, C.: Oscillatory magnetic flux tube slippage in the plasma sheet, Ann. Geophys., 24, 16951704, 2006, http://www.ann-geophys.net/24/1695/2006/.

Pritchett, P. L., Coronity, F. V., Pellat, R., and Karimabadi, H.: Collisionless reconnection in two-dimensional magnetic equilibria, J. Geophys. Res., 96, 11523-11538, 1991.

Runov, A., Sergeev, V. A., Baumjohann, W., Nakamura, R., Apatenkov, S., Asano, Y., Volwerk, M., Vörös, Z., Zhang, T. L., Petrukovich, A., Balogh, A., Sauvaud, J.-A., Klecker, B., and Rème, H.: Electric current and magnetic field geometry in flapping magnetotail current sheets, Ann. Geophys., 23, 1391-1403, 2005 , http://www.ann-geophys.net/23/1391/2005/.

Runov, A., Sergeev, V. A., Nakamura, R., Baumjohann, W., Zhang, T. L., Asano, Y., Volwerk, M., Vörös, Z., Balogh, A., and Réme, H.: Reconstruction of the magnetotail current sheet structure using multi-point Cluster measurements, Planet. Space Sci., 53, 237-243, 2005.

Runov, A., Sergeev, V. A., Nakamura, R., Baumjohann, W., Ap- 
atenkov, S., Asano, Y., Takada, T., Volwerk, M., Vörös, Z., Zhang, T. L., Sauvaud, J.-A., Rème, H., and Balogh, A.: Local structure of the magnetotail current sheet: 2001 Cluster observations, Ann. Geophys., 24, 247-262, 2006,

http://www.ann-geophys.net/24/247/2006/.

Sergeev, V., Runov, A., Baumjohann, W., Nakamura, R., Zhang, T. L., Volwerk, M., Balogh, A., Réme, H., Sauvaud, J. A., Andre, M., and Klecker, B.: Current sheet flapping motion and structure observed by Cluster, Geophys. Res. Lett., 30, 1327, doi:10.1029/2002GL016500, 2003.

Sergeev, V., Runov, A., Baumjohann, W., Nakamura, R., Zhang, T. L., Balogh, A., Louarn, P., Sauvaud, J.-A., and Réme, H.: Orientation and propagation of current sheet oscillations, Geophys. Res. Lett., 31, L05807, doi:10.1029/2003GL019346, 2004.

Sergeev, V. A., Sormakov, D. A., Apatenkov, S. V., Baumjohann, W., Nakamura, R., Runov, A. V., Mukai, T., and Nagai, T.: Survey of large-amplitude flapping motions in the midtail current sheet, Ann. Geophys., 24, 2015-2024, 2006,

http://www.ann-geophys.net/24/2015/2006/.
Schindler, K.: Physics of space plasma activity: Cambridge Univ. Press, Cambridge, 2007.

Sitnov, M. I., Swisdak, M., Drake, J. F., Guzdar, P. N., and Rogers, B. N.: A model of the bifurcated current sheet: 2. Flapping motion, Geophys. Res. Lett., 31, L09805, doi:10.1029/2004GL019473, 2004.

Smith, J. M., Roberts, B., and Oliver, R.: Magnetoacoustic wave propagation in current sheets, Astron. Astrophys., 327, 377-387, 1997.

Volwerk, M., Glassmeier, K.-H., Runov, A., Baumjohann, W., Nakamura, R., Zhang, T. L., Klecker, B., Balogh, A., and Réme, H.: Geophys. Res. Lett. 30(6), 1320, doi:10.1029/2002GL016467, 2003.

Zhang, S. T. L., Baumjohann, W., Nakamura, R., Balogh, A., and Glassmeier, K.-H.: A wavy twisted neutral sheet observed by Cluster, Geophys. Res. Lett., 29, 1899, doi:10.1029/2002GL015544, 2002. 